\title{
ITERATIONS CONVERGING TO DISTINCT SOLUTIONS OF SOME NONLINEAR OPERATOR EQUATIONS IN BANACH SPACE
}

\author{
IOANNIS K. ARGYROS \\ Department of Mathematics \\ University of Iowa \\ Iowa City, IA 52242
}

(Received April 29, 1985 and in revised form April 18, 1986)

ABSTRACT. We examine the solvability of multilinear equations of the form

$$
M_{k}(x, x, \ldots, x)=y, k=2,3, \ldots
$$

where $M_{k}$ is a $k$-linear operator on a Banach space $X$ and $y \in X$ is fixed.

KEY WORDS AND PHRASES. MUItilinear operator, contraction.

1980 AMS SUBJECT CLASSIFICATION CODE. $46 \mathrm{BI} 5$.

1. INTRODUCTION.

We study the quadratic equation

$$
B(x, x)=y
$$

in a Banach space $X$, where $B$ is a bounded symmetric bilinear operator on $X$ and $y$ is fixed in $X$ [2], [3], [7], [9], [10]. We consider two cases.

CASE 1. Let $y=0$ and set $x=\bar{x}-h$ for some $\bar{x}$ such that the linear operator $2 B(\bar{x})$ is invertible then (I.I) becomes

$$
\bar{B}(h, h)=h-\bar{y}
$$

where $\bar{B}=(2 B(\bar{x}))^{-1} B, \bar{y}=(2 B(\bar{x}))^{-1} B(\bar{x}, \bar{x})$ and $h \in X$ is to be determined.

We introduce the iteration

$$
h_{n+1}=\left(\bar{B}\left(h_{n}\right)\right)^{-1}\left(h_{n}-\bar{y}\right) \text { for some } h_{0} \in X
$$

to find a solution $h$ of (1.2) such that $h \neq \bar{x}$.

It turns out under certain assumptions that iteration (1.3) converges to an $h \in X$ such that $h \neq \bar{x}$, therefore $x=\bar{x}-h$ is a nonzero solution of (1.I). CASE 2. Let $y \neq 0$, we then introduce the iteration

$$
x_{n+1}=B\left(x_{n}\right)^{-1}(y) \text { for some } x_{0} \in \ddot{x}
$$

to find solutions of (1.1).

The results obtained here can be generalized to include multilinear equations of the form 


$$
\mathrm{N}_{\mathrm{k}}(\mathrm{x}, \mathrm{x}, \ldots, \mathrm{x})=\mathrm{y}
$$

where $M_{k}$ is a $k$-linear operator on $X$ and $y$ is fixed in $X$ [10].

We now state the following lemma. The proof can be found in [10].

2. EXISTENCE THEORY.

LEMMA 1. Let $L_{1}$ and $L_{2}$ be bounded linear operators in a Banach space $\lambda$, where $L_{1}$ is invertible, and $\left\|L_{1}^{-1}\right\| \cdot\left\|L_{2}\right\|<1$. Then $\left(L_{1}+I_{2}\right)^{-1}$ exists, amd

$$
\left\|\left(L_{1}+L_{2}\right)^{-1}\right\| \leq \frac{\left\|L_{1}^{-1}\right\|}{1-\left\|L_{2}\right\| \cdot\left\|L_{1}^{-1}\right\|} \text {. }
$$

LEMMA 2. Let $z \neq 0$ be fixed in $X$. Assume that the linear operator $\bar{B}(z)$ is invertible then $\bar{B}(x)$ is also invertible for all $x \in U(z, r)=\{x \in X \mid\|x-z\|<r\}$, where $r \in\left(0, r_{0}\right)$ and $r_{0}=\left[\|\bar{B}\| \cdot\left\|\bar{B}(z)^{-I}\right\|\right]^{-I}$.

PROOF. We have

$$
\begin{aligned}
\|\bar{B}(x-z)\| \cdot\left\|\bar{B}(z)^{-I}\right\| & \leq\|\bar{B}\| \cdot\|x-z\| \cdot\left\|\bar{B}(z)^{-I}\right\| \\
& \leq\|\bar{B}\| \cdot\left\|\bar{B}(z)^{-1}\right\| \cdot r \\
& <1
\end{aligned}
$$

for $r \in\left(0, r_{0}\right)$. The result now follows from Lemma 1 for $L_{1}=\bar{B}(z), L_{2}=\bar{B}(x-z)$

and $\mathrm{x} \in \mathrm{U}(\mathrm{z}, \mathrm{r})$.

DEFINITION 1. Assume that the linear operator $\overline{\mathrm{B}}(\mathrm{z})$ is invertible.

Define the operators $P, T$ on $U(z, r)$ for some $r>0$ by

$$
P(x)=\bar{B}(x, x)+\bar{y}-x, T(x)=(\bar{B}(x))^{-1}(x-\bar{y})
$$

and the real polynomials $f(r), g(r)$ on $R$ by

$$
\begin{aligned}
& f(r)=a^{\prime} r^{2}+b^{\prime} r+c^{\prime}, \quad g(r)=a r^{2}+b r+c, \\
& a^{\prime}=\left(\|\bar{B}\| \cdot\left\|\bar{B}(z)^{-1}\right\|\right)^{2}, \\
& b^{\prime}=-2\|\bar{B}\| \cdot\left\|\bar{B}(z)^{-1}\right\|, \\
& c^{\prime}=1-\left\|\bar{B}(z)^{-1}\right\|-\|\bar{B}\| \cdot\left\|\bar{B}(z)^{-1}\right\|^{2} \cdot\|z-\bar{y}\|, \\
& a \quad=\|\bar{B}\|\left\|\bar{B}(z)^{-1}\right\|, \\
& b \quad=\left\|\bar{B}(z)^{-1}(I-\bar{B}(z))\right\|-1,
\end{aligned}
$$

and

$$
c \quad=\left\|\bar{B}(z)^{-1} \mathrm{P}(\mathrm{z})\right\|
$$

THEOREM 1. Let $\mathrm{z} \in \mathrm{X}$ be such that $\overline{\mathrm{B}}(\mathrm{z})$ is invertible and that the following are true:

a) $c^{\prime}>0$;

b) $b<0, b^{2}-4 a c>0$, and

c) there exists $r>0$ such that $f(r)>0$ and $g(r) \leq 0$

then the iteration

$$
h_{n+1}=\bar{B}\left(n_{n}\right)^{-1}\left(h_{n}-\bar{y}\right), \quad n=0,1,2, \ldots
$$


is well defined and it converges to a unique solution $h$ of $(l . \hat{c})$ in $\bar{U}(z, r)$ for any $h_{0} \in \bar{U}(z, r)$.

PROOF. $T$ is well defined by Lemma 2 .

CLAIN 1. T maps $\bar{U}(z, r)$ into $\bar{U}(z, r)$.

If $x \in \bar{U}(z, r)$ then

$$
\begin{aligned}
T(x)-z & =\bar{B}(x)^{-1}(x-\bar{y})-z \\
& =\bar{B}(x)^{-1}[(I-\bar{B}(z))(x-z)-P(z)]
\end{aligned}
$$

so

$$
\|T(x)-z\| \leq r
$$

if

$$
\frac{1}{1-\|\bar{B}\| \cdot\left\|\bar{B}(z)^{-I}\right\| r}\left[\left\|\bar{B}(z)^{-I}(I-\bar{B}(z))\right\| r+\left\|\bar{B}(z)^{-I} P(z)\right\|\right] \leq r
$$

(using Lemma $I$ for $L_{1}=\bar{B}(z)$ and $L_{2}=\bar{B}(x-z)$ ) or $g(r) \leq 0$ which is true by hypothesis.

CLAIM 2. T is a contraction operator on $\bar{U}(z, r)$.

If $\mathrm{w}, \mathrm{v} \in \overline{\mathrm{U}}(\mathrm{z}, \mathrm{r})$ then

$\|\mathrm{T}(\mathrm{w})-\mathrm{T}(\mathrm{v})\|$

$=\left\|\bar{B}(w)^{-1}(w-\bar{y})-\bar{B}(v)^{-1}(v-\bar{y})\right\|$

$=\| \bar{B}(w)^{-1}\left[I-\bar{B}\left(\bar{B}(v)^{-1}(v-\bar{y})\right](w-v) \|\right.$

$\left.=\| \bar{B}(w)^{-1} \int I-\bar{B}\left(\bar{B}(v)^{-1}(v-z)\right)+\bar{B}\left(\bar{B}(v)^{-1}(z-\bar{y})\right)\right](w-v) \|$

$\leq \frac{1}{1-\|\bar{B}\| \cdot\left\|\bar{B}(z)^{-I}\right\| \cdot r}\left[\left\|\bar{B}(z)^{-1}\right\|+\| \frac{\|\bar{B}\| \cdot\left\|\bar{B}(z)^{-1}\right\|^{2} r+\|\bar{B}\|\left\|\bar{B}(z)^{-1}\right\|^{2}\|z-y\|}{1-\|\bar{B}\| \cdot\left\|\bar{B}(z)^{-1}\right\| \cdot r}\right] \cdot\|w-v\|$

$=q \cdot\|w-v\|$.

So $T$ is a contraction on $\bar{U}(z, r)$ if $0<q<1$, where

$$
q=\frac{1}{1-\|\bar{B}\| \cdot\left\|\bar{B}(z)^{-1}\right\| \cdot r}\left[\left\|\bar{B}(z)^{-1}\right\|+\frac{\|\bar{B}\| \cdot\left\|\bar{B}(z)^{-1}\right\|^{2} r+\|\bar{B}\| \cdot\left\|\bar{B}(z)^{-1}\right\|^{2}\|z-\bar{y}\|}{1-\|\bar{B}\| \cdot\left\|\bar{B}(z)^{-1}\right\| \cdot r}\right]
$$

which is true since $f(r)>0$.

THEOREM 2. Assume that there exist $r>0, z, \bar{x} \in X$ satisfying the hypotheses of Theorem $I$ and

$$
\begin{aligned}
& \text { (a) } 0<\|\bar{x}\|<-1+\frac{\sqrt{1+4\|\bar{B}\|\|\bar{y}\|}}{2\|\bar{B}\|} \text {; } \\
& \text { (b) } r+\|z\|<\frac{\|y\|}{1+\|\bar{B}\| \cdot\|\bar{x}\|} \text {; }
\end{aligned}
$$

then if $\|\bar{x}\|<h_{0} \leq r+\|z\|$, the solution $h$ if (1.2) is such that

$$
\|\bar{x}\|<\|h\| \leq r+\|z\| \text {. }
$$

Moreover, $x=\bar{x}-h$ is a nonzero solution of (1.1).

PROOF. By Theorem I $h \in \bar{U}(z, r)$ therefore

$$
\|h\| \leq r+\|z\| \text {. }
$$


Assume that $\left\|h_{k}\right\|>\|\bar{x}\|$ for $k=0,1,2, \ldots, n$. By iteration (1.3) we have

$$
\bar{B}\left(h_{n+1}, h_{n}\right)=h_{n}-\bar{y}
$$

or

$$
\|\bar{B}\|\left\|h_{n+1}\right\| \cdot\left\|h_{n}\right\| \geq\left\|h_{n}-\bar{y}\right\| \geq\|\bar{y}\|-\left\|h_{n}\right\|
$$

so

$$
\left\|h_{n+1}\right\| \geq \frac{\|\bar{y}\|-\left\|h_{n}\right\|}{\|\bar{B}\| \cdot\left\|h_{n}\right\|},
$$

to show that

$$
\left\|h_{n+1}\right\|>\|\bar{x}\|
$$

it suffices to show

$$
\frac{\|\bar{y}\|-\left\|h_{n}\right\|}{\|\bar{B}\|\left\|h_{n}\right\|}>\|\bar{x}\|
$$

which is true by (b). For consistency we must have

$$
\|\bar{x}\|<\frac{\|\bar{y}\|}{1+\|\bar{B}\| \cdot\|\bar{x}\|}
$$

which is true by (a). The result now follows by taking the limit as $n \rightarrow \infty$ in (2.1). Finally note that since $\|h\|>\|\bar{x}\|, \bar{x}-h \neq 0$ therefore $x=\bar{x}-h$ is a nonzero solution of (1.1).

DEFINITION 2. Assume that the linear operator $B(z)$ is invertible for some $z \in X$. Define the operator $\bar{P}$ on $U(z, r)$ for some $r>0$ by

$$
\bar{P}(x)=B(x, x)-y, \quad y \neq 0
$$

and the real polynomials $\bar{f}(r), \bar{g}(r)$ on $R$ by

where

$$
\bar{f}(r)=s_{1}^{\prime} r^{2}+s_{2}^{\prime} r+s_{3}^{\prime}, \quad \bar{g}(r)=s_{1} r^{2}+s_{2} r+s_{3},
$$

$$
\begin{aligned}
& s_{1}^{\prime}=\left(\|B\| \cdot\left\|B(z)^{-1}\right\|\right)^{2} \\
& s_{2}^{\prime}=-2\|B\| \cdot\left\|B(z)^{-1}\right\| \\
& s_{3}^{\prime}=1-\|B\| \cdot\left\|B(z)^{-1}\right\|^{2} \\
& s_{1}=\|B\| \cdot\left\|B(z)^{-1}\right\| \\
& s_{2}=\|B\| \\
& s_{3}=\left\|B(z)^{-1} \bar{P}(z)\right\| .
\end{aligned}
$$

The proofs of the following theorems are omitted as similar to Theorems 1 and 2 . THEOREM 3. Let $z \in X$ be such that the linear operator $B(z)$ is invertible and that the following are true:
a) $\mathrm{s}_{3}^{\prime}>0$
b) $s_{2}>0, s_{2}^{2}-4 s_{1} s_{2}>0$, and
c) there exists $r>0$ such that $\bar{f}(r)>0$ and $\bar{g}(r) \leq 0$ 
then the iteration

$$
x_{n+1}=B\left(x_{n}\right)^{-1}(y)
$$

for some $x_{0} \in X$ is well defined and it converges to a solution $x$ of (I.I) which is unique in $\bar{U}(z, r)$ for any $x_{0} \in \bar{U}(z, r)$.

THEOREM 4. Let $z, r$ be such that the hypotheses of Theorem 3 are satisfied.

Let $p<q$ be positive numbers such that

$$
\begin{aligned}
& \text { a) } p q\|B\| \leq\|y\| \text {; } \\
& \text { b) } \frac{\left\|E(z)^{-1}\right\|}{1-\|B\| \cdot\left\|B(z)^{-1}\right\| r} \leq q \leq r+\|z\|
\end{aligned}
$$

then if $p \leq\left\|x_{0}\right\| \leq q$ then the solution $x$ of (1.I) is such that

$$
p \leq\|x\| \leq q \text {. }
$$

\section{REFERENCES}

1. ANSELONE, P.M. and MOORE, R.H. An Extension of the Newton-Kantorovich Method for Solving Nonlinear Equations, Tech. Rep. No. 520, U.S. Army Mathematics Research Center, University of Wisconsin, Madison, 1965.

2. ARGYROS, I.K. On a Contraction Theorem and Applications, (to appear in "Proceedings of Symposia in Pure Mathematics" of the American Mathematical Society).

3. ARGYROS, I.K. Quadratic Equations in Banach Space, Perturbation Techniques and Applications to Chandrasekhar's and Related Equations, Ph.D. dissertation, University of Georgia, Athens, 1984.

4. BOWDEN, R.L. and ZWEIFEL, P.F. Astrophysics J. (1976), 219-232.

5. CHANDRASEKHAR, S. Radiative Transfer, Dover PubI., New York, 1960.

6. KELUY, C.T. Solution of the Chandrasekhar H Equation by Newton's Method, J. Math. Phys. 21 (1980), 1625-1628.

7. KELLEY, C.T. Approximation of Some Quadratic Integral Equations in Transport Theory, J. Integral Equations 4 (1982), 221-237.

8. MULLIKIN, T.W. Some Probability Distributions for Neutron Transport in HalfSpace, J. Applied Probability 2 (1968), 357-374.

9. RALL, L.B. Quadratic Equations in Banach Space, Rend. Circ. Math. Palermo 10 $(1961), 314-332$.

10. RALL, L.B. Computational Solution of Nonlinear Operator Equations, John Wiley Publ., New York, 1968.

11. STIBBS, D.W.N. and WEIR, R.E. On the H-functions for Isotropic Scattering, Monthly Not. Roy. Astron. Soc. 119 (1959), 515-525. 


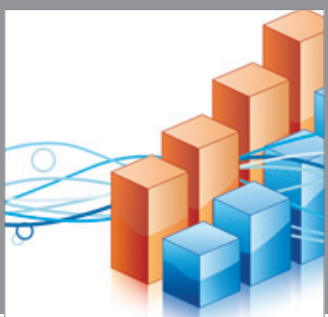

Advances in

Operations Research

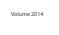

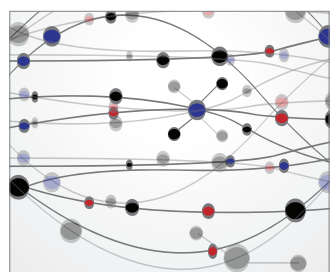

\section{The Scientific} World Journal
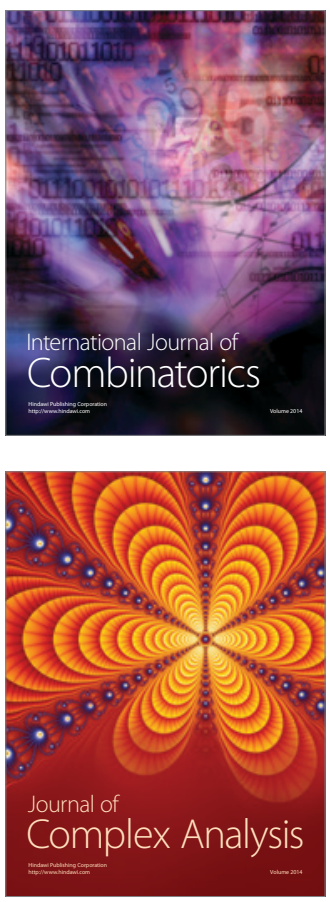

International Journal of

Mathematics and

Mathematical

Sciences
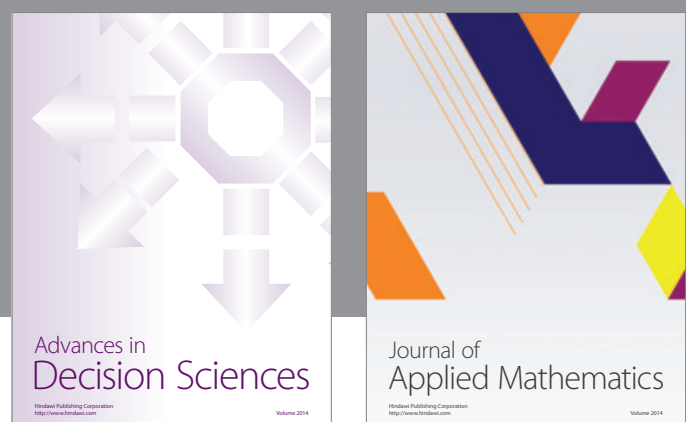

Journal of

Applied Mathematics
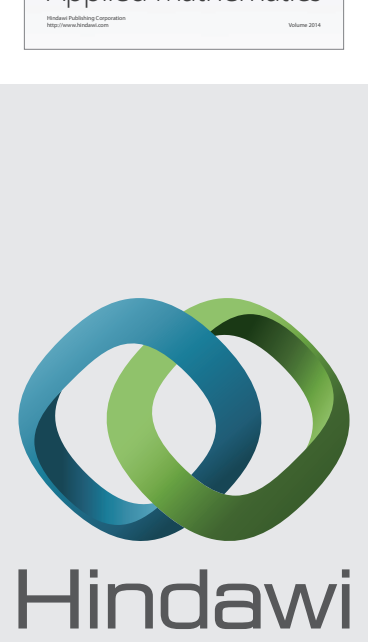

Submit your manuscripts at http://www.hindawi.com
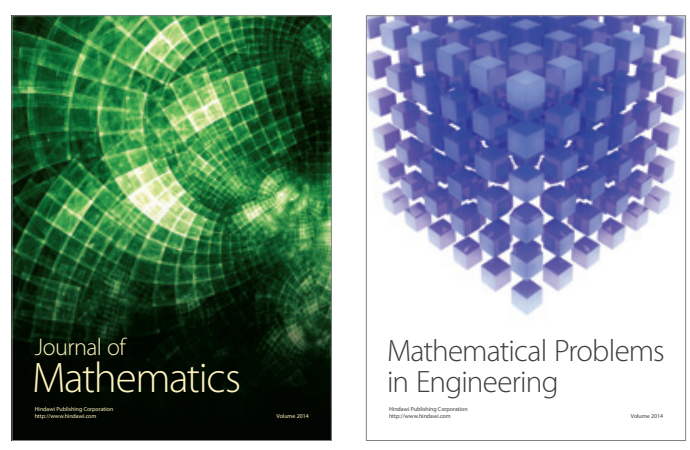

Mathematical Problems in Engineering
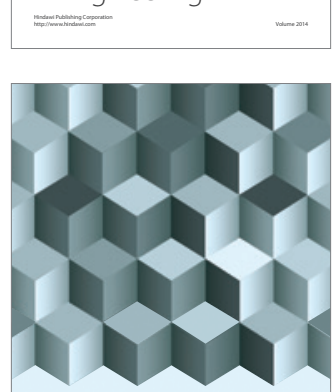

Journal of

Function Spaces
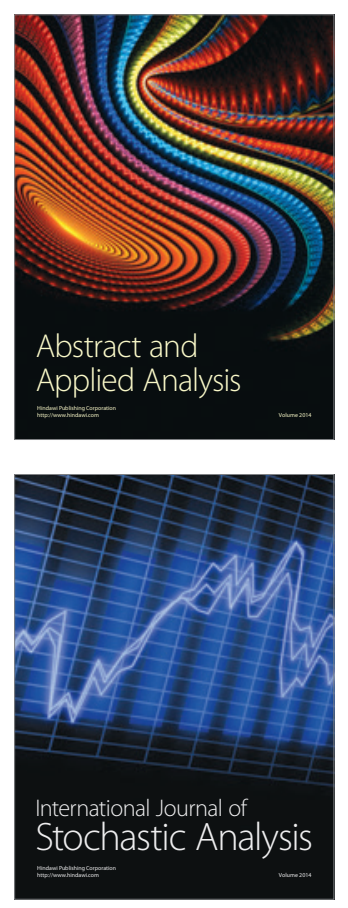

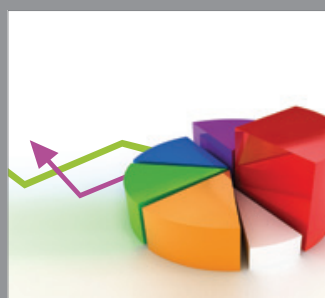

ournal of

Probability and Statistics

Promensencen
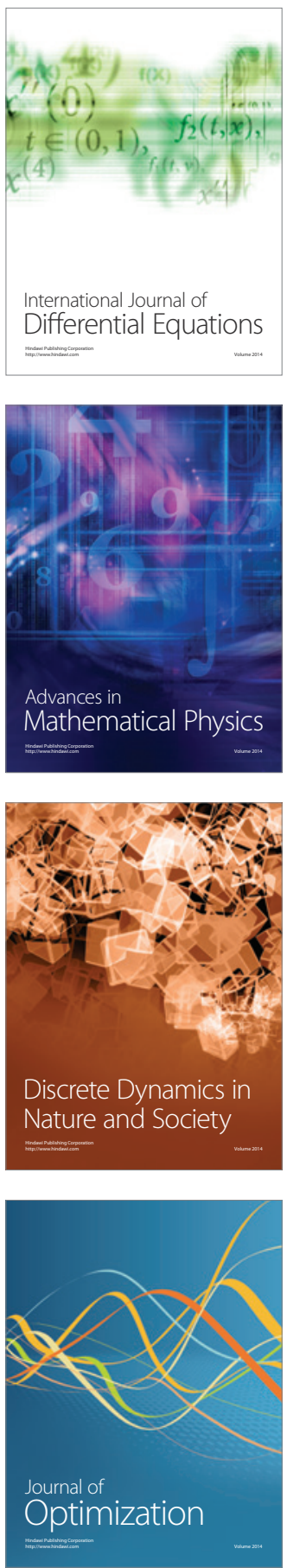